\title{
What Is the Ideal Tumor Regression Grading System in Rectal Cancer Patients after Preoperative Chemoradiotherapy?
}

\section{Soo Hee Kim, MD ${ }^{1, a}$ \\ Hee Jin Chang, MD, $P h D^{1,2}$ \\ Dae Yong Kim, MD, PhD2 \\ Ji Won Park, MD, PhD ${ }^{3}$ \\ Ji Yeon Baek, MD, PhD² \\ Sun Young Kim, MD, PhD² \\ Sung Chan Park, MD, MS² \\ Jae Hwan Oh, MD, PhD' \\ Ami Yu, PhD 4 \\ Byung-Ho Nam, PhD ${ }^{4}$}

\begin{abstract}
Purpose
Tumor regression grade (TRG) is predictive of therapeutic response in rectal cancer patients after chemoradiotherapy (CRT) followed by curative resection. However, various TRG systems have been suggested, with subjective categorization, resulting in interobserver variability. This study compared the prognostic validity of four different TRG systems in order to identify the most ideal TRG system.
\end{abstract}

\section{Materials and Methods}

This study included 933 patients who underwent preoperative CRT and curative resection. Primary tumors alone were graded according to the American Joint Committee on Cancer (AJCC), Dworak, and Ryan TRG systems, and both primary tumors and regional lymph nodes were graded according to a modified Dworak TRG system. The ability of each TRG system to predict recurrence-free survival (RFS) and overall survival (OS) was analyzed using chisquare and $\mathrm{C}$ statistics.

\section{Results}

All four TRG systems were significantly predictive of both RFS and OS ( $p<0.001$ each), however none was a better predictor of prognosis than ypStage. Among the four TRGs, the mDworak TRG system was a better predictor of RFS and OS than the AJCC, Dworak, and Ryan TRG systems, and both the chi-square and C statistics were higher for the former, although the differences were not statistically significant. The combination of ypStage and the modified Dworak TRG better predicted RFS and OS than ypStage alone.

\section{Conclusion}

The modified Dworak TRG system for evaluation of entire tumors including regional lymph nodes is a better predictor of survival than current TRG systems for evaluation of the primary tumor alone.
Correspondence: Hee Jin Chang, MD, PhD Department of Pathology,

Research Institute and Hospital, National Cancer Center, 323 Ilsan-ro, Ilsandong-gu, Goyang 10408 , Korea

Tel: $82-31-920-1741$

Fax: 82-31-920-1369

E-mail: heejincmd@yahoo.com

Received July 14, 2015

Accepted September 20, 2015

Published Online October 22, 2015

aPresent address: Anatomic Pathology Reference Lab, Seegene Medical Foundation, Seoul, Korea
Key words

Rectal neoplasms, Chemoradiotherapy, Tumor regression grade 


\section{Introduction}

Pre-operative chemoradiation therapy (CRT), followed by curative resection, has become the standard treatment for patients with locally advanced rectal cancer [1]. Accurate determination of tumor regression grade (TRG), ypT, ypN, and ypStage in the rectum after CRT is important for both pathologists and patients. TRG reflects therapeutic response, and ypT, ypN, and ypStage have been shown to predict prognosis [2-4]. The anatomical criteria in TNM staging are relatively objective and reproducible. Although the definition of regional lymph node (LN) metastasis (including pericolorectal tumor nodules) has been modified several times [5-7], ypN remains a major prognostic factor in these patients $[8,9]$. Various grading systems have been proposed for TRG, however, resulting in interobserver variability in grading [10]. The most widely used TRG systems are those of Ryan et al. [11], Dworak et al. [12], and Mandard [13]. The Mandard and Dworak TRG systems are classified according to five-point grades based on residual tumor and fibrosis [12,13], whereas the Ryan TRG system, with three-point grading, is a type of modified Mandard TRG system [11]. The 2010 American Joint Committee on Cancer (AJCC) TRG system is a modification of the Ryan TRG system based on the volume of residual primary tumor cells [5]. Details of each of these TRG systems are shown in Table 1.

The current TRG systems evaluate only the primary tumor with no consideration of regional LN status. To determine the most clinically-valid TRG system predictive of prognosis and therapeutic response, we retrospectively compared the prognostic significance of current TRG systems that evaluate the primary tumor alone with that of a newly developed modified Dworak (mDworak) TRG system that evaluates both the primary tumor and regional LNs.

\section{Materials and Methods}

\section{Patients}

This study enrolled 1,063 patients with primary rectal cancer who had undergone preoperative CRT at the National Cancer Center, Korea, between January 1, 2002 and December 30, 2011. All of the patients had biopsy-proven carcinoma of the middle or lower rectum (within $9 \mathrm{~cm}$ of the anal verge) and were classified as CT3 or CT4 on magnetic resonance imaging (MRI), with or without transrectal ultrasonography. Of these 1,063 patients, 130 were excluded, including 71 who refused surgery, 15 who were transferred to other hospitals,

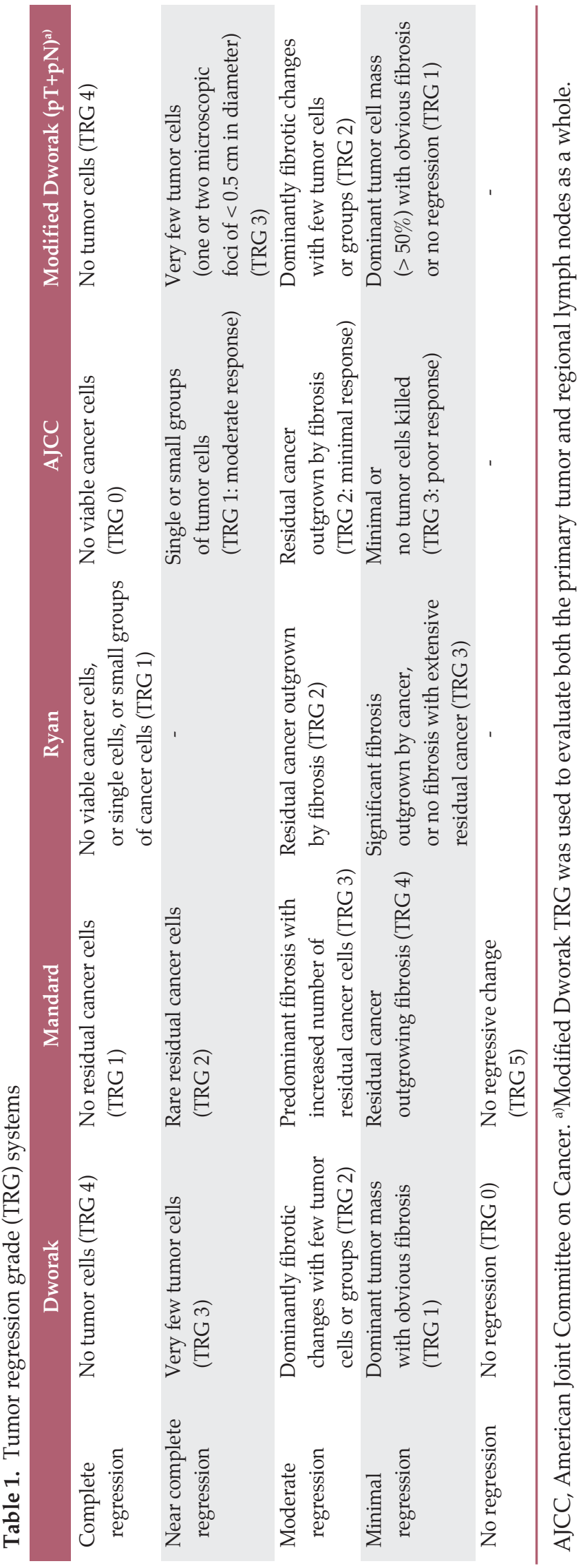


Table 2. Distribution of case numbers according to four different TRG systems

\begin{tabular}{lcccc} 
& \multicolumn{3}{c}{ Distribution } \\
\cline { 2 - 5 } TRG system & Modified Dworak TRG & AJCC TRG & Ryan TRG & Dworak TRG \\
Grade 0 & - & $135(14.5)^{\mathrm{a})}$ & - & 0 \\
Grade 1 & $162(17.3)$ & $140(15.0)$ & $275(29.5)$ & $113(12.1)$ \\
Grade 2 & $526(56.4)$ & $546(58.5)$ & $546(56.1)$ & $575(61.6)$ \\
Grade 3 & $116(12.4)$ & $112(12.0)$ & $112(11.5)$ & $110(11.8)$ \\
Grade 4 & $129(13.8)$ & - & - & $135(14.5)$ \\
\hline
\end{tabular}

Values are presented as number $(\%)$. The modified Dworak system assessed the primary tumor and regional lymph nodes, whereas the American Joint Committee on Cancer (AJCC), Ryan, and Dworak systems assessed the primary tumor alone.

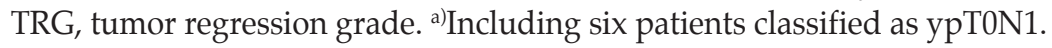

and 44 who underwent local excision because of the presence of comorbidities or inoperable status (initial clinical stage IV). The remaining 933 patients were treated with neoadjuvant CRT, followed by curative resection. Neoadjuvant CRT consisted of preoperative radiotherapy (total dose, 45 Gy) applied over 5-6 weeks to the pelvis, with a boost to the rectum, resulting in a total of $50.4 \mathrm{~Gy}$ in 28 fractions. Concomitant chemotherapy was initiated on the first day of radiotherapy, and administered intravenously or orally during the 6 weeks of radiotherapy. Multiple chemotherapeutic regimens were employed, with 536 patients $(57.5 \%)$ treated with 5-fluorouracil (5-FU) and leucovorin; 255 (27.3\%) with capecitabine, with or without irinotecan; $117(12.5 \%)$ with tegafur-uracil; and 25 (2.7\%) with cetuximab, irinotecan, and capecitabine. Radical surgery, including total mesorectal excision, was performed 4-6 weeks after completion of CRT. Of the 944 patients, $809(86.7 \%)$ subsequently received adjuvant chemotherapy, consisting of fluoropyrimidine (5-FU/leucovorin, capecitabine, or tegafur-uracil/leucovorin; $\mathrm{n}=747$ ) or combination therapy (5-FU/leucovorin/ oxaliplatin, capecitabine/oxaliplatin, S-1/oxaliplatin, or 5-FU/leucovorin/irinotecan; $\mathrm{n}=62$ ). This study was approved by the Institutional Review Board of the National Cancer Center, Korea, and each patient provided written informed consent prior to preoperative CRT.

\section{Pathological examination}

Each tumor was classified using World Health Organization (WHO) criteria [14] and initially staged using the TNM system of the AJCC, sixth edition [6]. TRGs for both primary tumors and regional LNs were initially determined using our mDworak TRG system. The original Dworak TRG system evaluates the primary tumor only [12], whereas our mDworak TRG system evaluates the entire lesion including the rectum and underlying mesorectum. The resected rectum was embedded in full thickness for evaluation of the circum- ferential resection margin and total mesorectal excision status. Sections from the rectal wall often include the regional LNs and perirectal tumor deposits. TRG determination may be ambiguous in patients having predominant residual cancer cells in the mesorectum as a separate nodular form (regressed mesorectal tumor [ypT3] vs. perirectal tumor deposits [ypN1c]). Thus, we estimated TRG for the primary tumor and regional LNs, including perirectal tumor deposits, as a whole. The mDworak TRG system was graded as follows: TRG 4, or complete regression, defined as no residual tumor cells in the primary tumor and regional LNs (ypT0N0); TRG 3, or near complete regression, defined as one or two microscopic foci (each $<0.5 \mathrm{~cm}$ in diameter) of residual tumor cells or groups in the primary tumor and regional LNs; TRG 2, or moderate regression, defined as dominant fibroinflammatory changes with vasculopathy encompassing more than $50 \%$ of the entire tumor, including the tumor, regional LN metastases, and perirectal tumor deposits; TRG 1, or minimal regression, defined as a dominant tumor mass encompassing more than $50 \%$ of the primary tumor and/or regional LN metastases. All tumors were reviewed by two pathologists (S.H.K. and H.J.C.) to determine tumor deposit status (ypN1c); this allowed restaging of the tumors according to the seventh edition of the AJCC [5]. TRGs of the primary tumors were also re-assessed using the Dworak, Ryan, and AJCC TRG systems [5,11,12]. Since the Mandard and Dworak TRG systems have similar grading criteria, with the only difference being the reverse order of TRG number (Table 1), tumor assessment using the Mandard TRG system was not performed. Of the 933 patients, $55(5.89 \%)$ changed their TRGs by using Dworak TRG instead of the mDworak TRG system. Among the 55 patients, six were found to be ypT0N+ (ypT0N1), with these patients classified as having complete regression according to the AJCC and Dworak TRG systems. Patient distribution according to each TRG system is shown in Table 2. 


\section{Follow-up}

Patients were followed-up for local recurrence and distant metastasis every 3 months for the first 2 postoperative years, then every 6-12 months thereafter. Follow-up included physical examinations, measurements of serum carcinoembryonic antigen concentration, chest X-ray, and abdominal ultrasound or computed tomography (CT).

\section{Statistical analysis}

Pearson's chi-square test or Fisher exact test was used for comparison of between-group differences in recurrence or survival rate predicted by clinicopathological parameters. Overall survival (OS) was defined as the time from diagnosis to death. Relapse-free survival (RFS) was defined as the time from operation to any type of recurrence, as evidenced by CT, MRI, or histology. RFS and OS curves were plotted using the Kaplan-Meier method and compared using the log-rank test. Prognostic factors were evaluated using Cox regression models. The predictive abilities of the mDworak, AJCC, original Dworak (Dworak), and Ryan TRG systems, and ypStage for RFS and OS were evaluated by chi-square and C statistics, the latter being a concordance measure analogous to the receiver operating characteristic curve area for the logistic model. The value indicates the probability that a model produces a higher risk for those who do than do not develop an event [15]. Higher chi-square and C-statistic values indicate better predictive capabilities. Models that combined ypStage with each TRG were also examined. Interobserver variation of mDworak TRG systems was also analyzed by kappa value. A p-value of $<0.05$ was considered statistically significant. Statistical analyses were performed using IBM SPSS ver. 20 software for Windows (IBM Co., Armonk, NY), and the chi-square and C statistics were calculated using Stata software (Stata Corp., College Station, TX).

\section{Results}

\section{Patients}

Of the 933 patients, 188 (20.1\%) experienced tumor recurrence over a median follow-up period of 53.7 months (range, 0 to 126 months). Locoregional recurrence occurred in 101 patients $(10.8 \%)$, distant metastasis in $171(18.3 \%)$, of whom $84(9 \%)$ had both locoregional recurrence and distant metastasis. The 5-year RFS and OS rates were $77.4 \%$ and $62.8 \%$, respectively. The number of patients in each TRG classification is shown in Table 2. Over 50\% of patients graded using the mDworak, Ryan, Dworak and AJCC TRGs were grade 2. The clinicopathologic characteristics of the included patients are shown in Table 3.

\section{Survival analysis (RFS and OS)}

In Kaplan-Meier univariate analysis, ypN, ypT, and ypStage; all four TRG systems (mDworak, AJCC, Dworak, and Ryan); histological grade; venous, lymphatic, and perineural invasion; and circumferential resection margin showed significant association with both RFS and OS ( $p<0.01$ each) (Table 3). The RFS and OS of patients classified as original or mDworak grades 3 and 4, and those classified as AJCC TRG0 and 1, did not differ significantly (Table 3, Figs. 1 and 2). The RFS and OS of ypStage 0 and I patients also did not differ significantly ( $p>0.1$ each) (Table 3). RFS was significantly lower in the ypT0N+ (ypT0N1) than in other early stage groups $(\mathrm{p}<0.001)$ (Fig. 1). The 5-year RFS rate of the ypT0N+ group was comparable to that of the ypstage III group (55.6\% vs. $58 \%$, respectively) (Table 3). Two of six ypT0N+ patients developed recurrences at 20 and 51 months postoperatively, and both were classified as ypT0N1a. However, no ypT0N+ patient died during the follow-up period.

Using multivariate analysis, we performed an analysis to determine whether each TRG system, as well as ypStage, histological grade, perineural invasion, and circumferential resection margin, were prognostic of RFS and OS. In multivariate analysis, only ypStage, perineural invasion, and circumferential resection margin were independently prognostic for RFS and OS (Table 4).

\section{Ability of the four TRG systems and ypStage to predict RFS and OS}

The chi-square and $C$ statistics of a model using ypStage for prediction of RFS and OS were significantly higher than those of the models using the TRG systems, indicating that ypStage was a better predictor of RFS and OS than the TRG systems (Table 5). Among the four TRGs, the mDworak TRG system was a better predictor of RFS and OS than the AJCC, Dworak, and Ryan TRG systems, and both the chi-square and $\mathrm{C}$ statistics were higher for the former, although the differences were not statistically significant (Table 5). However, the combination of ypStage and the mDworak TRG system showed significantly better chi-square and $C$ statistics for both RFS and OS than ypStage alone (Table 5). A combination of ypStage and the AJCC TRG system showed increased chi-square and $\mathrm{C}$ statistics for RFS and OS compared with ypStage alone; however, this model did not distinguish among the hazard ratios of groups ( $p>0.05$ for AJCC TRG), indicating that this model was inadequate for prognosis. 

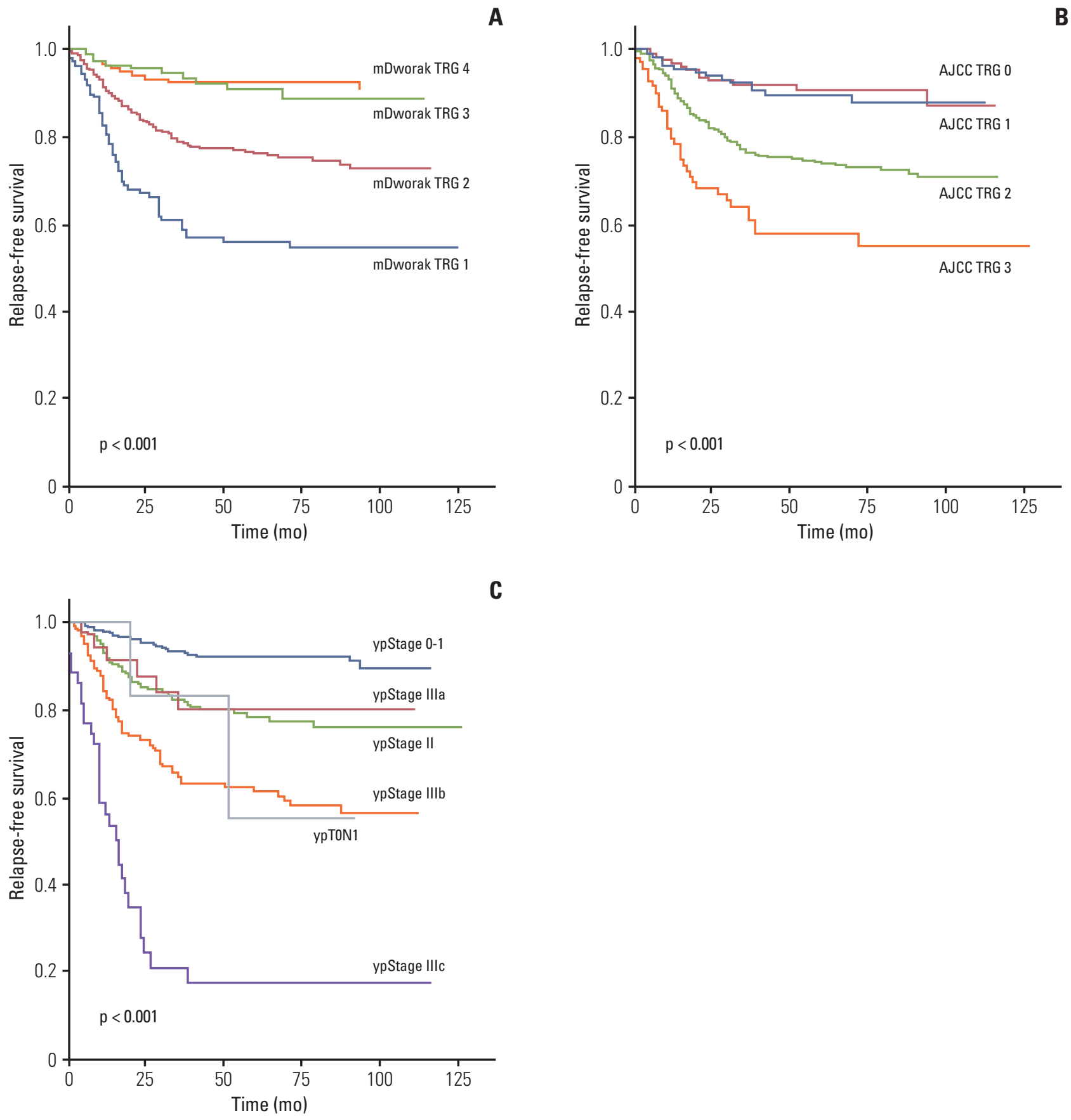

Fig. 1. Relapse-free survival of 933 rectal cancer patients treated with pre-operative chemoradiotherapy followed by surgical resection, according to tumor regression grades (TRG) according to the modified Dworak (mDworak) system (A), which assesses both the primary tumor and regional lymph nodes, the American Joint Committee on Cancer (AJCC) system (B), which assesses the primary tumor alone, and ypStage (C). 

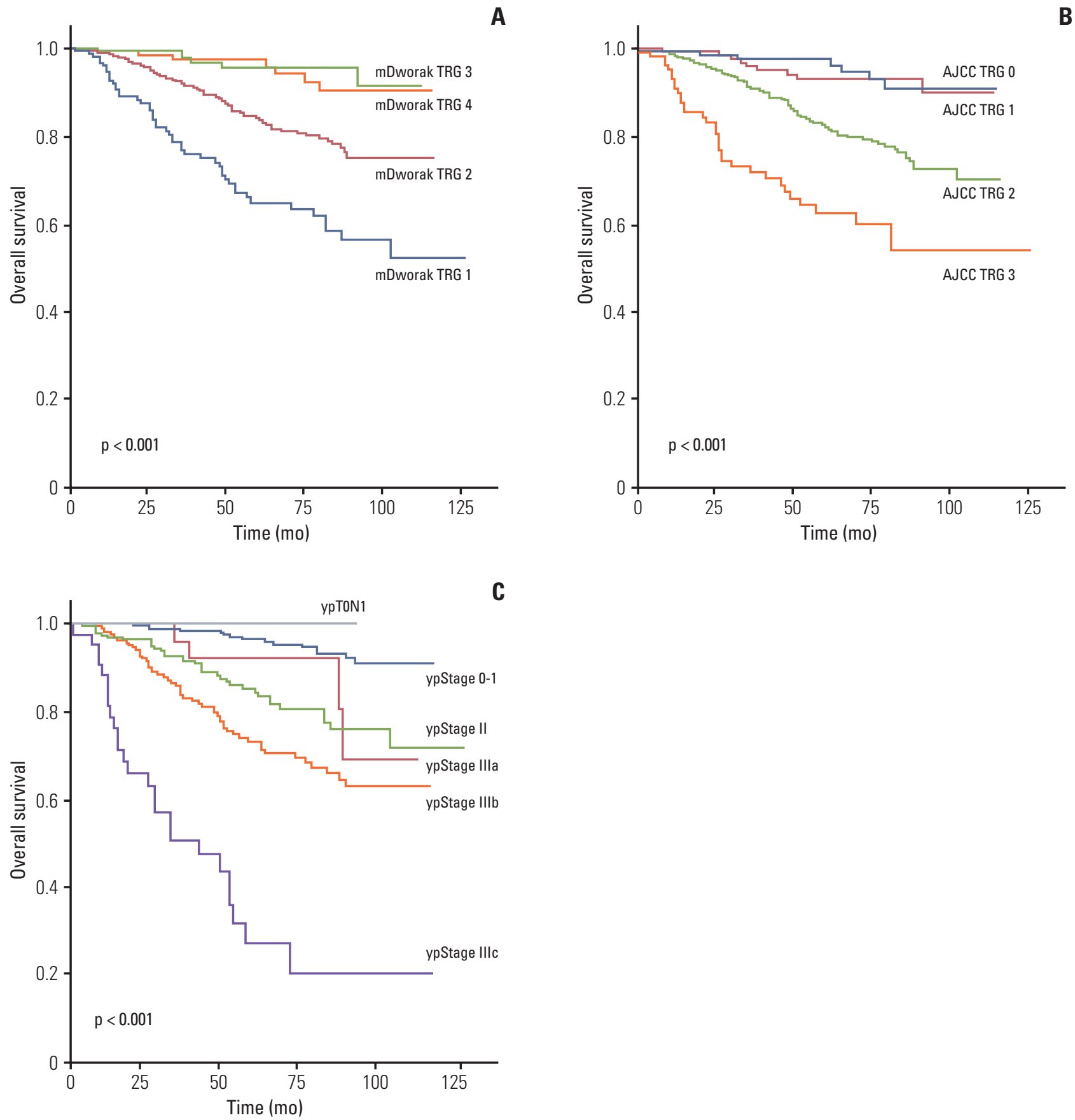

Fig. 2. Overall survival of 933 rectal cancer patients treated with pre-operative chemoradiotherapy followed by surgical resection, according to tumor regression grades (TRG) according to the modified Dworak (mDworak) system (A), which assesses both the primary tumor and regional lymph nodes, the American Joint Committee on Cancer (AJCC) system (B), which assesses the primary tumor alone, and ypStage (C). 
Table 3. Parameters used in Kaplan-Meier survival analysis

\begin{tabular}{|c|c|c|c|c|c|}
\hline Parameter & No. of cases & 5-Year RFS (\%) & p-value & 5-Year OS (\%) & p-value \\
\hline \multicolumn{6}{|l|}{ Sex } \\
\hline Male & 635 & 76.4 & 0.434 & 98.4 & 0.271 \\
\hline Female & 298 & 78.7 & & 95.3 & \\
\hline \multicolumn{6}{|l|}{ Age $(y r)^{a)}$} \\
\hline$<60$ & 530 & 77.2 & 0.887 & 86.2 & 0.057 \\
\hline$\geq 60$ & 403 & 77.6 & & 97.1 & \\
\hline \multicolumn{6}{|c|}{ Distance from $\mathrm{AV}(\mathrm{cm})$} \\
\hline$<5$ & 318 & 71.3 & 0.002 & 81.6 & 0.15 \\
\hline$\geq 5$ & 615 & 80.8 & & 85.9 & \\
\hline \multicolumn{6}{|l|}{ Histological type ${ }^{\text {b) }}$} \\
\hline Adenocarcinoma & 768 & 75.5 & $<0.001$ & 83.1 & $<0.001$ \\
\hline Other type ${ }^{c)}$ & 31 & 60.9 & & 57.5 & \\
\hline \multicolumn{6}{|l|}{ Histological grade $^{\text {b) }}$} \\
\hline Low & 757 & 76 & $<0.001$ & 83.6 & $<0.001$ \\
\hline High & 42 & 56.1 & & 54.5 & \\
\hline \multicolumn{6}{|l|}{ ypT } \\
\hline урт0 & 134 & 92 & $<0.001$ & 97.6 & $<0.001$ \\
\hline ypTis & 12 & 91.7 & & 87.5 & \\
\hline ypT1 & 51 & 97.8 & & 97.3 & \\
\hline урT2 & 222 & 88.7 & & 95.4 & \\
\hline урT3 & 468 & 66.9 & & 73.9 & \\
\hline урT4 & 46 & 55.1 & & 73.6 & \\
\hline \multicolumn{6}{|l|}{ ypN } \\
\hline ypN0 & 612 & 87 & $<0.001$ & 92.1 & $<0.001$ \\
\hline ypN1a & 83 & 68.1 & & 82.4 & \\
\hline ypN1b & 103 & 64.6 & & 77.3 & \\
\hline ypN1c & 33 & 59 & & 65.1 & \\
\hline ypN2a & 61 & 45.4 & & 60.4 & \\
\hline ypN2b & 41 & 22.4 & & 29.7 & \\
\hline \multicolumn{6}{|l|}{ ypStage } \\
\hline ypT0N1 & 6 & 55.6 & $<0.001$ & 100 & $<0.001$ \\
\hline 0 & 140 & 93.1 & & 97.7 & \\
\hline I & 228 & 91.6 & & 96 & \\
\hline II & 244 & 78.5 & & 83.6 & \\
\hline III & 315 & 58 & & 68.9 & \\
\hline IV & 0 & 0 & & 0 & \\
\hline \multicolumn{6}{|l|}{ Circumferential RM } \\
\hline Negative & 847 & 80 & $<0.001$ & 87.8 & $<0.001$ \\
\hline Positive & 86 & 48.8 & & 52 & \\
\hline \multicolumn{6}{|l|}{ mDworak TRG ${ }^{\text {d) }}$} \\
\hline 1 (minimal) & 162 & 56 & $<0.001$ & 64.9 & $<0.001$ \\
\hline 2 (moderate) & 526 & 76.2 & & 83.7 & \\
\hline 3 (near complete) & 116 & 91.1 & & 95.8 & \\
\hline 4 (compete) & 129 & 92.5 & & 97.5 & \\
\hline \multicolumn{6}{|l|}{ AJCC TRG } \\
\hline 0 (complete) & 135 & 90.9 & $<0.001$ & 97.6 & $<0.001$ \\
\hline 1 (moderate) & 140 & 89.7 & & 93 & \\
\hline 2 (minimal) & 546 & 73.9 & & 82.2 & \\
\hline 3 (poor) & 112 & 57.8 & & 62.4 & \\
\hline
\end{tabular}


Table 3. Continued

\begin{tabular}{|c|c|c|c|c|c|}
\hline Parameter & No. of cases & 5-Year RFS (\%) & p-value & 5-Year OS (\%) & p-value \\
\hline \multicolumn{6}{|l|}{ Dworak TRG } \\
\hline$\leq 1$ (minimal) & 113 & 57.1 & $<0.001$ & 62.6 & $<0.001$ \\
\hline 2 (moderate) & 575 & 74.3 & & 82.3 & \\
\hline 3 (near complete) & 110 & 93 & & 95.6 & \\
\hline 4 (complete) & 135 & 90.9 & & 97.6 & \\
\hline \multicolumn{6}{|l|}{ Ryan TRG } \\
\hline 1 (good) & 275 & 90.3 & $<0.001$ & 95.2 & $<0.001$ \\
\hline 2 (moderate) & 546 & 73.9 & & 82.2 & \\
\hline 3 (poor) & 112 & 57.8 & & 62.4 & \\
\hline \multicolumn{6}{|l|}{ TME } \\
\hline Complete & 664 & 79.2 & 0.072 & 85.7 & 0.275 \\
\hline Near-complete & 236 & 73 & & 81 & \\
\hline Incomplete & 33 & 64.1 & & 71.3 & \\
\hline \multicolumn{6}{|l|}{ Lymphatic invasion } \\
\hline Present & 256 & 58.4 & $<0.001$ & 63.9 & $<0.001$ \\
\hline Absent & 677 & 83.8 & & 90.9 & \\
\hline \multicolumn{6}{|l|}{ Perineural invasion } \\
\hline Present & 215 & 79.8 & $<0.001$ & 62.1 & $<0.001$ \\
\hline Absent & 718 & 84.7 & & 89.9 & \\
\hline \multicolumn{6}{|l|}{ Venous invasion } \\
\hline Present & 185 & 57.6 & $<0.001$ & 62.1 & $<0.001$ \\
\hline Absent & 748 & 96.7 & & 88.7 & \\
\hline
\end{tabular}

RFS, recurrence-free survival; OS, overall survival; AV, anal verge; RM, resection margin; mDwork, modified Dwork; TRG,

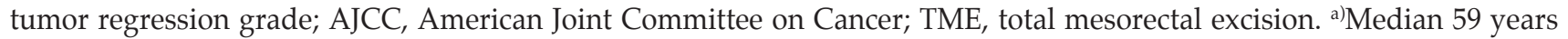
(range, 22 to 87 years), b)No residual tumors were noted in 134 cases (14.4\%); these are excluded, c) 23 mucinous adenocarcinomas, six signet ring cell carcinomas, two adenosquamous carcinomas, ${ }^{\mathrm{d}}$ Dworak TRG assessing primary tumor and regional lymph nodes as a whole.

Table 4. Multivariate analysis of factors influencing RFS and OS

\begin{tabular}{|c|c|c|c|c|}
\hline \multirow{2}{*}{ Factor } & \multicolumn{2}{|l|}{ RFS } & \multicolumn{2}{|l|}{ OS } \\
\hline & Hazard ratio $(95 \% \mathrm{CI})$ & p-value & Hazard ratio $(95 \% \mathrm{CI})$ & p-value \\
\hline \multicolumn{5}{|l|}{ ypStage } \\
\hline $0 \& \mathrm{I}$ & 1.000 & $<0.001$ & 1.000 & $<0.001$ \\
\hline II & $2.057(1.253-3.379)$ & & $2.668(1.449-4.913)$ & \\
\hline III & $4.514(2.888-7.055)$ & & $4.747(2.686-8.389)$ & \\
\hline \multicolumn{5}{|c|}{ Perineural invasion } \\
\hline Absent & 1.000 & $<0.001$ & 1.000 & $<0.001$ \\
\hline Present & $2.440(1.802-3.304)$ & & $2.161(1.504-3.105)$ & \\
\hline \multicolumn{5}{|c|}{ Circumferential resection margin } \\
\hline Negative & 1.000 & 0.010 & 1.000 & $<0.001$ \\
\hline Positive & $1.656(1.128-2.430)$ & & $2.942(1.979-4.375)$ & \\
\hline
\end{tabular}

RFS, recurrence-free survival; OS, overall survival; CI, confidence interval. 
Table 5. Univariate Cox's proportional hazards models and model validation of RFS and OS

\begin{tabular}{|c|c|c|c|c|c|c|c|c|}
\hline \multirow{2}{*}{ Model } & \multicolumn{4}{|c|}{ RFS } & \multicolumn{4}{|c|}{ OS } \\
\hline & HR $(95 \%$ CI) & p-value & $X^{2}$ & Harrell's Ca) & HR $(95 \%$ CI) & p-value & $x^{2}$ & Harrell's $C^{b)}$ \\
\hline Modified Dworak TRG & & & 68.92 & 0.6492 & & & 58.06 & 0.6783 \\
\hline 1 & 1.000 & & & & 1.000 & & & \\
\hline 2 & $0.450(0.336-0.603)$ & $<0.001$ & & & $0.426(0.297-0.610)$ & $<0.001$ & & \\
\hline 3 & $0.178(0.099-0.322)$ & $<0.001$ & & & $0.106(0.042-0.267)$ & $<0.001$ & & \\
\hline 4 & $0.172(0.097-0.305)$ & $<0.001$ & & & $0.132(0.060-0.292)$ & $<0.001$ & & \\
\hline AJCC TRG & & & 59.58 & 0.6359 & & & 53.95 & 0.6718 \\
\hline 0 & 1.000 & & & & 1.000 & & & \\
\hline 1 & $1.035(0.523-2.048)$ & 0.922 & & & $1.243(0.463-3.337)$ & 0.666 & & \\
\hline 2 & $2.662(1.587-4.464)$ & $<0.001$ & & & $3.696(1.710-7.986)$ & 0.001 & & \\
\hline 3 & 5.553 (3.146-9.803) & $<0.001$ & & & $9.036(4.004-20.394)$ & $<0.001$ & & \\
\hline Dworak TRG & & & 61.85 & 0.6374 & & & 55.52 & 0.6711 \\
\hline$\leq 1$ & 1.000 & & & & 1.000 & & & \\
\hline 2 & $0.460(0.332-0.637)$ & $<0.001$ & & & $0.403(0.272-0.599)$ & $<0.001$ & & \\
\hline 3 & $0.149(0.077-0.287)$ & $<0.001$ & & & $0.098(0.038-0.250)$ & $<0.001$ & & \\
\hline 4 & $0.177(0.101-0.312)$ & $<0.001$ & & & $0.111(0.049-0.251)$ & $<0.001$ & & \\
\hline Ryan TRG & & & 59.57 & 0.6356 & & & 53.76 & 0.6700 \\
\hline 1 & 1.000 & & & & 1.000 & & & \\
\hline 2 & $2.615(1.791-3.820)$ & $<0.001$ & & & 3.289 (1.929-5.610) & $<0.001$ & & \\
\hline 3 & $5.457(3.492-8.527)$ & $<0.001$ & & & $8.043(4.437-14.580)$ & $<0.001$ & & \\
\hline ypStage & & & 119.46 & 0.7046 & & & 82.46 & 0.7175 \\
\hline$\leq \mathrm{I}$ & 1.000 & & & & 1.000 & & & \\
\hline II & $2.892(1.885-4.436)$ & $<0.001$ & & & $3.959(2.191-7.154)$ & $<0.001$ & & \\
\hline $\mathrm{III}^{\mathrm{c})}$ & $6.328(4.339-9.229)$ & $<0.001$ & & & $7.864(4.608-13.422)$ & $<0.001$ & & \\
\hline ypStage & & & 133.35 & 0.7248 & & & 97.33 & 0.7482 \\
\hline$\leq \mathrm{I}$ & 1.000 & & & & 1.000 & & & \\
\hline II & $2.613(1.532-4.456)$ & $<0.001$ & & & $3.297(1.551-7.007)$ & 0.002 & & \\
\hline $\mathrm{III}^{\mathrm{c})}$ & $5.404(3.286-8.886)$ & $<0.001$ & & & $6.206(3.040-12.669)$ & $<0.001$ & & \\
\hline \multicolumn{9}{|l|}{ Modified Dworak TRG } \\
\hline 1 & 1.000 & & & & 1.000 & & & \\
\hline 2 & $0.612(0.455-0.824)$ & 0.001 & & & $0.57(0.395-0.822)$ & 0.003 & & \\
\hline 3 & $0.411(0.22-0.769)$ & 0.005 & & & $0.25(0.097-0.649)$ & 0.004 & & \\
\hline 4 & $0.702(0.333-1.480)$ & 0.352 & & & $0.639(0.223-1.827)$ & 0.403 & & \\
\hline ypStage & & & 130.76 & 0.7208 & & & 96.72 & 0.7439 \\
\hline$\leq \mathrm{I}$ & 1.000 & & & & 1.000 & & & \\
\hline II & $2.600(1.567-4.315)$ & $<0.001$ & & & 3.141 (1.569-6.285) & 0.001 & & \\
\hline $\mathrm{III}^{\mathrm{c})}$ & $5.439(3.438-8.606)$ & $<0.001$ & & & $5.949(3.146-11.249)$ & $<0.001$ & & \\
\hline \multicolumn{9}{|l|}{ AJCC TRG } \\
\hline 0 & 1.000 & & & & 1.000 & & & \\
\hline 1 & $0.710(0.352-1.435)$ & 0.340 & & & $0.766(0.276-2.216)$ & 0.608 & & \\
\hline 2 & $0.983(0.532-1.816)$ & 0.955 & & & $1.203(0.490-2.956)$ & 0.687 & & \\
\hline 3 & $1.654(0.848-3.226)$ & 0.140 & & & $2.464(0.957-6.347)$ & 0.062 & & \\
\hline
\end{tabular}

RFS, recurrence-free survival; OS, overall survival; HR, hazard ratio; CI, confidence interval; TRG, tumor regression grade;

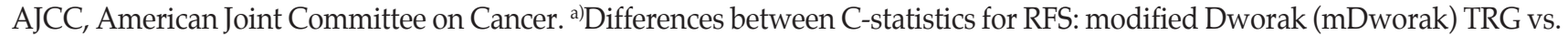
AJCC TRG, $\mathrm{p}=0.091$; $\mathrm{mDw}$ orak TRG vs. Dworak TRG, $\mathrm{p}=0.118$; mDworak TRG vs. Ryan TRG, $\mathrm{p}=0.110$; AJCC TRG vs. Dworak TRG, $\mathrm{p}=0.794$; AJCC TRG vs. Ryan TRG, $\mathrm{p}=0.893$; Dworak TRG vs. Ryan TRG, $\mathrm{p}=0.750$; $y$ pStage vs. $\mathrm{mDworak}$ TRG, $\mathrm{p}<0.001$;

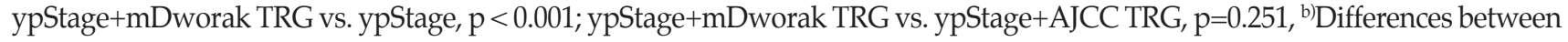
C-statistics for OS: $m$ Dworak TRG vs. AJCC TRG, $\mathrm{p}=0.542$; $\mathrm{mDw}$ worak TRG vs. Dworak TRG, $\mathrm{p}=0.407$; mDworak TRG vs. Ryan TRG, $\mathrm{p}=0.444$; AJCC TRG vs. Dworak TRG, $\mathrm{p}=0.925$; AJCC TRG vs. Ryan TRG, $\mathrm{p}=0.475$; Dworak TRG vs. Ryan TRG, $\mathrm{p}=0.878$; ypStage vs. mDworak TRG, $\mathrm{p}=0.043$; ypStage $+\mathrm{mDworak}$ TRG vs. ypStage, $\mathrm{p}<0.001$; ypStage+mDworak TRG vs. ypStage + AJCC TRG, $\mathrm{p}=0.582$, ${ }^{\mathrm{c}}$ Including six patients classified as ypT0N1. 


\section{Discussion}

An ideal TRG system should consistently measure therapeutic response and predict patient outcomes. However, previous studies on the prognostic significance of current TRG systems have yielded variable results, owing to the use of different grading systems, different endpoints for pathological complete response, different TRG components, and/or ambiguous grading criteria [10,16-18]. In addition, classification according to TRG systems showed a very low concordance rate among experienced gastrointestinal pathologists, even when using the same TRG system, indicating poor reproducibility of these systems [10]. Current TRG systems have two major limitations: the subjectivity of the grading criteria and the range of tumors being evaluated (i.e., the primary tumor alone or the primary tumor and regional LNs).

The endpoint of pathologic complete response has been defined as ypT0N0 $[19,20]$; however, current TRG systems evaluate only the primary tumor $[5,11,12]$. Even though regional $\mathrm{LN}$ status after CRT (ypN) is the most important prognostic factor, current TRG systems do not consider regional LN metastasis. Thus, the TRG systems may be inaccurate in predicting prognosis, particularly in ypT0N+ patients. Even though ypT0N+ patients have residual tumors, they would be classified as having achieved complete response using the current TRG systems. RFS and OS rates were significantly lower in ypT0N+ patients than in ypT0N0 patients [21]. This study therefore compared the predictive abilities of four TRG systems: the Dworak, Ryan, AJCC, and mDworak TRG systems. Although all were predictive of OS and RFS, the mDworak TRG system, which assesses both the primary tumor and regional LNs, was superior to the other TRG systems, which assess the primary tumor alone. However, none of the four TRG systems was superior in predictive ability to ypStage, but the mDworak TRG system was found to complement the predictive power of ypStage, further suggesting that consideration of regional LN status could enhance the prognostic ability of TRG systems that evaluate the primary tumor alone.

Another limitation of current TRG systems is that grading is imprecise and the criteria, particularly for near complete regression, may be very subjective. For example, Dworak TRG 3 was originally defined as 'very few (difficult to find microscopically) tumor cells in fibrotic tissue with or without mucous substance' [12]; however, this criterion was modified to 'regression of $>50 \%$ of the tumor mass' $[22,23]$. The latter criterion was actually for 'good regression' of the five-point TRG system proposed by Rodel et al. [18]. Similarly, Mandard TRG 2 was originally defined as 'the presence of rare residual cancer cells scattered throughout areas of fibrosis' [13], but has been modified to 'single cells, or small groups of cancer cells' in the Ryan TRG system [11]. The meaning of 'small groups of cancer cells' was further modified from near complete regression to moderate regression in the AJCC TRG system [5]. To overcome the subjectivity of these criteria, it may be necessary to quantify the estimated volume of residual tumor cells, perhaps by assessing the modified rectal cancer regression grade (m-RCRG). The criteria for $\mathrm{m}-\mathrm{RCRG}$ are grade 1 (complete or near-complete regression), defined as no tumor epithelium and scattered foci of malignant epithelium comprising $<5 \%$ of the overall area of abnormality; grade 2 (moderate regression), defined as malignant epithelium comprising $5 \%-50 \%$ of the overall area of abnormality; and grade 3 (minimal regression), defined as malignant epithelium comprising $>50 \%$ of the area of abnormality [10]. The m-RCRG is a quantified version of the Ryan TRG, except that one of the criteria of $\mathrm{m}$-RCRG grade $1,<5 \%$ of the residual tumor lesion,' may not match 'near-complete regression' of large tumors. The total residual tumor cell volume may vary by tumor size or number of sections. Therefore, 'nearcomplete regression' (grade 3) of the mDworak TRG system was defined as one or two microscopic foci $(<0.5 \mathrm{~cm}$ in diameter) of residual tumor cells or groups of tumor cells in the primary tumor and regional LNs. The criteria for moderate regression could consequently be determined by the criteria for near-complete and minimal regression. The mDworak TRG system defined the upper limit of moderate regression as $50 \%$ of residual tumor cell volume within the regressed tumor lesion.

A major limitation of this study was the heterogeneity in chemotherapeutic regimens. Use of different combination regimens may have affected therapeutic responses, the results of TRGs, and patient prognosis $[19,24]$. However, this limitation may not have had a significant impact in comparative analysis of TRG systems. The other limitation was that we did not compare interobserver variability among the various TRG systems. However, in random analysis of $5 \%$ of our cases (47 cases) for interobserver variability, kappa value for mDworal TRG between two pathologists (S.H.K. and H.J.C.) was 0.936 (data not shown). This kappa value is much higher than those reported in the previous study [10], and the reason why may be due to the differences in numbers of observers (2 vs. 17), and due to microscopic examination of entire lesions instead of one representative digitalized image. In addition the grading criteria of the mDworak TRG system could be relatively objective.

Despite multiple trials of various TRG systems, none was found to be a better predictor of prognosis than ypStage. Pathologic staging after neoadjuvant therapy is more objective and more predictive of prognosis and therapeutic responses (for complete response vs. partial response). Pathologic evaluation of surgically resected specimens after neoadjuvant therapy is an extra-burden for pathologists, 
since meticulous examination is necessary for the accurate evaluation of pathologic stage and therapeutic responses [25]. Thus, application of a clinically valid TRG system is necessary. Our results showed that the mDworak TRG system may complement ypStage, with their combination better predictive of RFS and OS than ypStage alone.

\section{Conclusion}

In conclusion, an ideal TRG system should reflect the therapeutic responses of both the primary tumor and regional LNs, and the criteria should not be subjective. Our mDworak TRG system may be an example of an ideal TRG system, enabling better prediction of survival, either alone or in combination with ypStage.

\section{Conflicts of Interest}

Conflict of interest relevant to this article was not reported.

\section{Acknowledgments}

This study was supported by the Converging Research Center Program funded by the Ministry of Science, ICT and Future Planning, Republic of Korea (Project No. 2013K000 271).

The authors thank Mr. Dong-Su Jang, Research Assistant, Department of Anatomy, Yonsei University College of Medicine, Seoul, Korea, for his help with the figures. We also thank Ms. Kyung-Min Kang, Biometric Research Branch, Research Institute and Hospital, National Cancer Center, Goyang, Korea, for her help with the statistical analysis.

\section{References}

1. Quah HM, Chou JF, Gonen M, Shia J, Schrag D, Saltz LB, et al. Pathologic stage is most prognostic of disease-free survival in locally advanced rectal cancer patients after preoperative chemoradiation. Cancer. 2008;113:57-64.

2. Hermanek P, Merkel S, Hohenberger W. Prognosis of rectal carcinoma after multimodal treatment: ypTNM classification and tumor regression grading are essential. Anticancer Res. 2013;33:559-66.

3. Kim DW, Kim DY, Kim TH, Jung KH, Chang HJ, Sohn DK, et al. Is T classification still correlated with lymph node status after preoperative chemoradiotherapy for rectal cancer? Cancer. 2006;106:1694-700.

4. Vecchio FM, Valentini V, Minsky BD, Padula GD, Venkatraman ES, Balducci $\mathrm{M}$, et al. The relationship of pathologic tumor regression grade (TRG) and outcomes after preoperative therapy in rectal cancer. Int J Radiat Oncol Biol Phys. 2005; 62:752-60.

5. Edge SB, Byrd DR, Compton CC, Fritz AG, Greene FL, Trotti A 3rd. AJCC cancer staging manual. 7th ed. New York: Springer-Verlag; 2010.

6. Greene FL, Page DL, Fleming ID, Fritz AG, Balch CM, Haller DG, et al. AJCC cancer staging manual. 6th ed. New York: Springer-Verlag; 2002.

7. Fleming ID, Cooper JS, Henson DE, Hutter RV, Kennedy BJ, Murphy GP, et al. AJCC cancer staging manual. 5th ed. Philadelphia, PA: Lippincott-Raven Publishers; 1997.

8. Lee SD, Kim TH, Kim DY, Baek JY, Kim SY, Chang HJ, et al. Lymph node ratio is an independent prognostic factor in patients with rectal cancer treated with preoperative chemora- diotherapy and curative resection. Eur J Surg Oncol. 2012; 38:478-83.

9. Tsai CJ, Crane CH, Skibber JM, Rodriguez-Bigas MA, Chang GJ, Feig BW, et al. Number of lymph nodes examined and prognosis among pathologically lymph node-negative patients after preoperative chemoradiation therapy for rectal adenocarcinoma. Cancer. 2011;117:3713-22.

10. Chetty R, Gill P, Govender D, Bateman A, Chang HJ, Deshpande $\mathrm{V}$, et al. International study group on rectal cancer regression grading: interobserver variability with commonly used regression grading systems. Hum Pathol. 2012;43: 1917-23.

11. Ryan R, Gibbons D, Hyland JM, Treanor D, White A, Mulcahy $\mathrm{HE}$, et al. Pathological response following long-course neoadjuvant chemoradiotherapy for locally advanced rectal cancer. Histopathology. 2005;47:141-6.

12. Dworak O, Keilholz L, Hoffmann A. Pathological features of rectal cancer after preoperative radiochemotherapy. Int J Colorectal Dis. 1997;12:19-23.

13. Mandard AM, Dalibard F, Mandard JC, Marnay J, HenryAmar M, Petiot JF, et al. Pathologic assessment of tumor regression after preoperative chemoradiotherapy of esophageal carcinoma: clinicopathologic correlations. Cancer. 1994; 73:2680-6.

14. Bosman FT, Carneiro F, Hruban RH. WHO classification of tumours of the digestive system. 4 th ed. Lyon: IARC Press; 2010.

15. Balakrishnan N, Rao CR. Handbook of statistics: advances in survival analysis. Burlington, MA: Elsevier; 2004. 
16. Rullier A, Laurent C, Capdepont M, Vendrely V, Bioulac-Sage $\mathrm{P}$, Rullier E. Impact of tumor response on survival after radiochemotherapy in locally advanced rectal carcinoma. Am J Surg Pathol. 2010;34:562-8.

17. Suarez J, Vera R, Balen E, Gomez M, Arias F, Lera JM, et al. Pathologic response assessed by Mandard grade is a better prognostic factor than down staging for disease-free survival after preoperative radiochemotherapy for advanced rectal cancer. Colorectal Dis. 2008;10:563-8.

18. Rodel C, Martus P, Papadoupolos T, Fuzesi L, Klimpfinger M, Fietkau R, et al. Prognostic significance of tumor regression after preoperative chemoradiotherapy for rectal cancer. J Clin Oncol. 2005;23:8688-96.

19. Rodel C, Liersch T, Becker H, Fietkau R, Hohenberger W, Hothorn T, et al. Preoperative chemoradiotherapy and postoperative chemotherapy with fluorouracil and oxaliplatin versus fluorouracil alone in locally advanced rectal cancer: initial results of the German CAO/ARO/AIO-04 randomised phase 3 trial. Lancet Oncol. 2012;13:679-87.

20. Maas M, Nelemans PJ, Valentini V, Crane CH, Capirci C, Rodel C, et al. Adjuvant chemotherapy in rectal cancer: defining subgroups who may benefit after neoadjuvant chemoradiation and resection: a pooled analysis of 3,313 patients. Int J Cancer. 2015;137:212-20.
21. Yeo SG, Kim DY, Kim TH, Chang HJ, Oh JH, Park W, et al. Pathologic complete response of primary tumor following preoperative chemoradiotherapy for locally advanced rectal cancer: long-term outcomes and prognostic significance of pathologic nodal status (KROG 09-01). Ann Surg. 2010; 252:998-1004.

22. Huebner M, Wolff BG, Smyrk TC, Aakre J, Larson DW. Partial pathologic response and nodal status as most significant prognostic factors for advanced rectal cancer treated with preoperative chemoradiotherapy. World J Surg. 2012;36:675-83.

23. Trakarnsanga A, Gonen M, Shia J, Nash GM, Temple LK, Guillem JG, et al. Comparison of tumor regression grade systems for locally advanced rectal cancer after multimodality treatment. J Natl Cancer Inst. 2014;106:dju248.

24. Hong YS, Nam BH, Kim KP, Kim JE, Park SJ, Park YS, et al. Oxaliplatin, fluorouracil, and leucovorin versus fluorouracil and leucovorin as adjuvant chemotherapy for locally advanced rectal cancer after preoperative chemoradiotherapy (ADORE): an open-label, multicentre, phase 2, randomised controlled trial. Lancet Oncol. 2014;15:1245-53.

25. Park SY, Chang HJ, Kim DY, Jung KH, Kim SY, Park JW, et al. Is step section necessary for determination of complete pathological response in rectal cancer patients treated with preoperative chemoradiotherapy? Histopathology. 2011;59:650-9. 